City University of New York (CUNY)

CUNY Academic Works

2015

\title{
Variation in Rheumatoid Hand and Wrist Surgery among Medicare Beneficiaries: A Population-based Cohort Study
}

\author{
Lin Zhong \\ University of Michigan-Ann Arbor \\ Kevin C. Chung \\ University of Michigan-Ann Arbor \\ Onur Baser \\ University of Michigan-Ann Arbor \\ David A. Fox \\ University of Michigan-Ann Arbor \\ Huseyin Yuce \\ CUNY New York City College of Technology
}

See next page for additional authors

\section{How does access to this work benefit you? Let us know!}

More information about this work at: https://academicworks.cuny.edu/ny_pubs/457

Discover additional works at: https://academicworks.cuny.edu

This work is made publicly available by the City University of New York (CUNY).

Contact: AcademicWorks@cuny.edu 
Authors

Lin Zhong, Kevin C. Chung, Onur Baser, David A. Fox, Huseyin Yuce, and Jennifer F. Waljee 


\title{
Variation in Rheumatoid Hand and Wrist Surgery among Medicare Beneficiaries: A Population-based Cohort Study
}

\author{
Lin Zhong, Kevin C. Chung, Onur Baser, David A. Fox, Huseyin Yuce, and Jennifer F. Waljee
}

\begin{abstract}
Objective. To examine the rate and variation in rheumatoid arthritis (RA)-related hand and wrist surgery among Medicare (elderly) beneficiaries in the United States, and to identify the patient and provider factors that influence surgical rates.

Methods. Using the 2006-2010 100\% Medicare claims data of beneficiaries with RA diagnosis, we examined rates of rheumatoid hand and wrist arthroplasty, arthrodesis, and hand tendon reconstruction in the United States. We used multivariate logistic regression models to examine variation in receipt of surgery by patient and regional characteristics (density of providers, intensity of use of biologic disease-modifying antirheumatic drugs).

Results. Between 2006 and 2010, the annual rate of RA-related hand and wrist arthroplasty or arthrodesis was 23.1 per 10,000 patients, and the annual rate of hand tendon reconstruction was 4.2 per 10,000 patients. The rates of surgery varied 9-fold across hospital referral regions in the United States. Younger patient age, female sex, white race, higher socioeconomic status (SES), and rural residence were associated with a higher likelihood of undergoing arthroplasty and arthrodesis. We observed a significant decline in rate of arthroplasty and arthrodesis with increasing density of rheumatologists. Tendon reconstruction was not influenced by provider factors, but was correlated with age, race, SES, and rural status of the patients.

Conclusion. Surgical reconstruction of rheumatoid hand deformities varies widely across the United States, driven by both regional availability of subspecialty care in rheumatology and individual patient factors. (First Release Jan 15 2015; J Rheumatol 2015;42:429-36; doi:10.3899/jrheum.140658)
\end{abstract}

Key Indexing Terms:

RHEUMATOID ARTHRITIS HAND WRIST SURGERY MEDICARE ELDERLY

Rheumatoid arthritis (RA) is a chronic, debilitating, immune-mediated disease involving progressive joint destruction, commonly affecting the small joints of the hand and wrist. Unfortunately, the incidence and prevalence of

From the Department of Surgery, Section of Plastic Surgery, Department of Internal Medicine, and Division of Rheumatology, University of Michigan Medical School, University of Michigan Health System, Ann Arbor, Michigan; and the Department of Mathematics, New York City College of Technology, New York, New York, USA.

Supported in part by a grant from the US National Institute of Arthritis and Musculoskeletal and Skin Diseases (R01 AR047328), and a 2013 Clinical Research Grant funded by the American Foundation for Surgery of the Hand (to Dr. Waljee).

L. Zhong, MD, MPH, Clinical Research Coordinator; K.C. Chung, MD, MS, Professor of Surgery, Assistant Dean for Faculty Affairs, Department of Surgery, Section of Plastic Surgery; O. Baser, PhD, Adjunct Professor, Department of Internal Medicine, University of Michigan Medical School; D.A. Fox, MD, Professor, Department of Internal Medicine, Division Chief, Division of Rheumatology; J.F. Waljee, MD, MS, Assistant Professor, Department of Surgery, Section of Plastic Surgery, University of Michigan Health System; H. Yuce, PhD, Associate Professor, Department of Mathematics, New York City College of Technology.

Address correspondence to Dr. J.F. Waljee, Section of Plastic Surgery, University of Michigan Health System, 2130 Taubman Center, SPC 5340, 1500 E. Medical Center Drive, Ann Arbor, Michigan 48109-5340, USA. E-mail: filip@med.umich.edu

Accepted for publication October 29, 2014.
$\mathrm{RA}$ are rising among older individuals ${ }^{1,2}$. Although there is no cure, surgical intervention, such as joint arthrodesis and arthroplasty, can improve pain and deformity for patients with RA, and many patients report improved satisfaction and independence in daily life $\mathrm{l}^{3,4,5}$.

Nonetheless, the role of surgery for RA remains controversial. Many clinicians view surgery as a failure of medical management, and prospective cohort studies in several countries suggest a declining rate of RA-related joint procedures $^{6,7,8,9}$, likely attributable to advances in medical therapy ${ }^{10}$. In the United States, rheumatologists and surgeons debate the effectiveness, timing, and indications for surgery, and studies have demonstrated large area variation in the rate of hand surgery among patients with $\mathrm{RA}^{11,12}$. Given this uncertainty, rates of surgery may represent systematic differences in access to appropriate, high-quality subspecialty care.

Currently, the factors that are correlated with undergoing RA-related hand and wrist surgery are unknown, but could reveal differences in access to care, physician practice patterns, and treatment effectiveness. In this context, we examined the rates of surgical procedures performed for rheumatoid hand and wrist deformities among all Medicare

Personal non-commercial use only. The Journal of Rheumatology Copyright @ 2015 . All rights reserved. 
(elderly) beneficiaries diagnosed with RA from 2006 to 2010 to (1) define the national variation in RA-related hand and wrist surgery, and (2) identify the patient and regional factors that influenced this variation. We hypothesized that measurable variation existed in the rates of surgery among elderly patients with rheumatoid hand and wrist deformities because of both patient and regional factors.

\section{MATERIALS AND METHODS}

Study sample and data source. The study sample was drawn from $100 \%$ Medicare beneficiaries who were diagnosed with RA and enrolled in fee-for-service programs between 2006 and 2010. To increase the reliability of the diagnosis of RA, the study cohort included beneficiaries with at least 2 diagnoses of RA [International Classification of Diseases, 9th ed (ICD-9) codes for RA: 714.0, 714.1, 714.2, 714.3, 714.4] listed > 7 and < 365 days apart between 2006 and $2010^{13}$. Additionally, we included beneficiaries aged $\geq 65$ years who were continuously enrolled in Medicare Part A and B during each calendar year. We excluded all beneficiaries who were enrolled in Medicare Advantage Plans (Part C) because of the lack of outpatient claims data in the Centers of Medicare and Medicaid Services (CMS) databases. Using residence postal code, we further limited our analysis to beneficiaries who resided within the 50 states and District of Columbia during the study period. Each study subject entered the cohort in the year of first diagnosis of RA, and remained in the cohort until the year of death, disenrollment, or end of our study.

We specifically reviewed all outpatient services for the RA cohort during the study period drawn from the outpatient files and the carrier files. We obtained demographic data and confirmed enrollment status using the Annual Beneficiary Summary Files. The outpatient files contained all fee-for-service claims submitted by outpatient providers, and the carrier files contained claims submitted by noninstitutional providers such as physicians, physician assistants, and nurse practitioners. Both files use ICD-9 codes to detail diagnosis, and Current Procedural Terminology (CPT) codes to detail procedures.

Dependent variables. Our outcome variables included RA-related hand and wrist surgical procedures identified by CPT codes (Table 1). Arthrodesis procedures included carporadial, carpometacarpal (CMC), metacarpophalangeal (MCP), and interphalangeal (IP) joint arthrodesis. Arthroplasty procedures included reconstruction with or without implants of the MCP, IP, CMC, intercarpal, and wrist joints. We also included tendon recon- structive procedures, specifically tenolysis, extensor tendon repair, and tendon transfers.

Patient factors in independent variables. We examined the characteristics of each study subject at their entry into the study cohort. We specifically included age (categorized as 65-69 yrs, 70-74 yrs, 75-80 yrs, and $\geq 80$ yrs), race (white, African American, and other), sex, and socioeconomic status (SES). SES was estimated using postal code of beneficiary residence and then grouped into 3 categories (high, medium, and low) ${ }^{14}$. As an additional measure of SES, we examined whether each beneficiary received state assistance in Medicare premiums using the "Medicare State Buy-in" variable in the Beneficiary Summary File. We identified these recipients if the beneficiary received the assistance for more than 6 months in the year entering the cohort. Additionally, we controlled for the overall health condition of study subjects in our analysis using Elixhauser comorbidity index ${ }^{15}$. Finally, we determined rural and urban residence for each subject using the 1993 US Department of Agriculture Rural-Urban Continuum Codes for Metro and Non-metro Counties ${ }^{16}$. Counties were labeled by their Federal Information Processing Standards (FIPS) codes and categorized into metropolitan counties (codes 1-3), non-metropolitan counties with more than 2500 urban population (codes 4-7), and completely rural counties (codes 8-9) in this database. We used the SAS 9.2 postal code help file, containing crosslink of postal code and FIPS code for each county in the United States, to link the FIPS code in the Rural-Urban Continuum Codes file to the residence postal code of the study cohort, to assign rural status for each patient.

Regional factors. Hospital referral regions (HRR) were defined by the Dartmouth Atlas of Health Care and represented regional healthcare markets for tertiary medical care, including 306 distinct regions in the United States according to patterns of hospital use ${ }^{17}$. We assigned each subject in the study cohort to an HRR by postal code of permanent residence regardless of where the procedure was performed for the patient.

To understand the effect of regional availability of specialist care, we obtained density of rheumatologists, orthopedic surgeons, and plastic surgeons within each HRR. This was detailed in the Dartmouth Atlas of Health Care as the number of specialists per 1 million residents per region. We then categorized provider density into evenly distributed tertiles.

To understand the effect of regional patterns of medical therapy, we calculated the rate of use of biologic disease-modifying antirheumatic drugs (DMARD) by each HRR. We identified use of biologic DMARD from Medicare outpatient claims and Medicare Part D Prescription Drug events by Healthcare Common Procedure codes and generic name of drugs

Table 1. RA-related procedures of the hand and wrist.

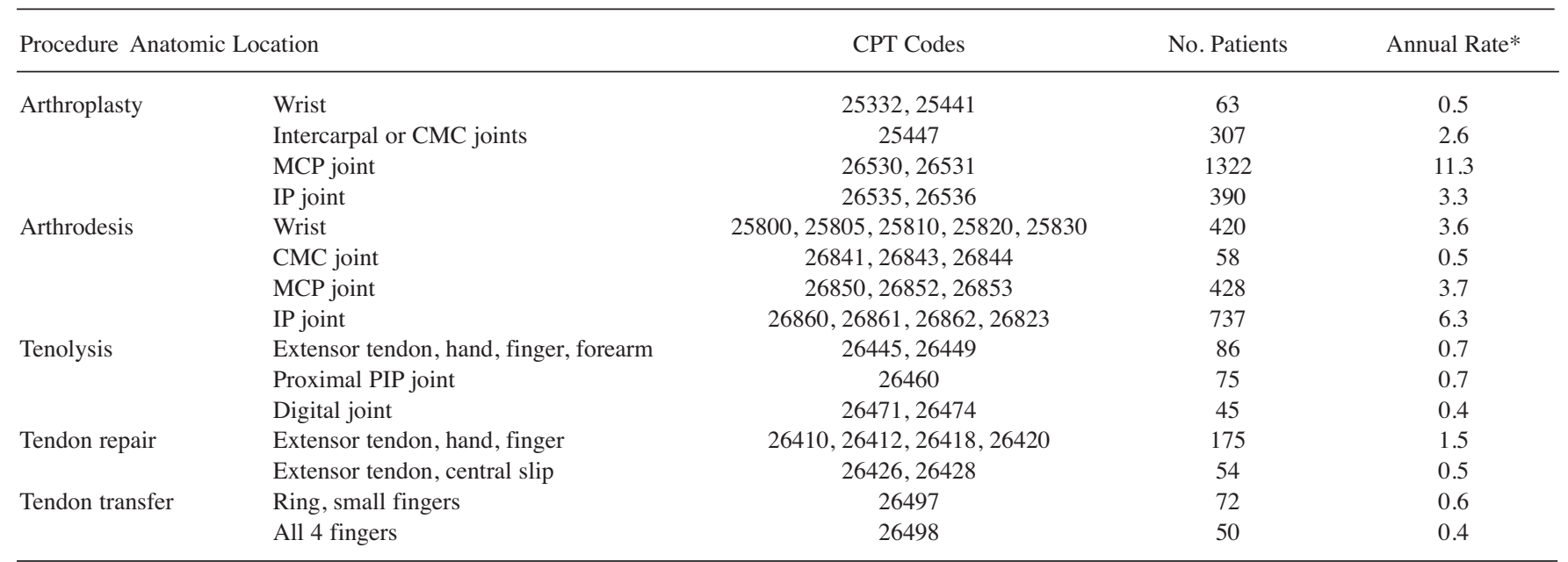

* Annual rate was calculated as no. patients with RA who received the procedure per 10,000 patients per year. RA: rheumatoid arthritis; CPT: Current Procedural Terminology; PIP: proximal interphalangeal; CMC: carpometacarpal; MCP: metacarpophalangeal; IP: interphalangeal. 
(Appendix 1). We calculated the annual rate of biologic DMARD use for each HRR as average percent of patients with RA who received biologic DMARD from 2006 to 2010.

Statistical analysis. We used descriptive statistics to detail the overall rates of RA-related hand and wrist surgery, and the sociodemographic characteristics of the study population. We then constructed multivariate logistic regression models to understand the effect of patient and regional factors on RA-related hand and wrist surgery. To examine the influence of regional availability of specialists on RA-related surgery, we also included in the model the density of orthopedic surgeons, plastic surgeons, and rheumatologists in the region where each study subject resided, as predictors of receiving RA surgery. Finally, we adjusted each model for number of years in the study cohort and regional density of biologics therapy.

\section{RESULTS}

In our cohort of Medicare beneficiaries, we identified 356,061 individuals diagnosed with RA (Table 2). In this cohort, 2895 patients underwent RA-related hand and wrist reconstructive surgery during the period (annual rate $=$ 24.8/10,000 patients). Among patients who underwent surgery, 2693 had hand or wrist arthroplasty or arthrodesis (annual rate $=23.1 / 10,000$ patients) and 488 had tendon reconstruction (annual rate $=4 \cdot 2 / 10,000$ patients). $\mathrm{MCP}$ joint arthroplasty was the most commonly performed surgery (annual rate $=11.3 / 10,000$ patients), followed by interphalangeal joint arthrodesis (annual rate $=6.3 / 10,000$ patients). Among tendon reconstruction procedures, extensor tendon repair was most commonly performed (annual rate $=1.5 / 10,000$ patients; Table 1$)$.

Figure 1 demonstrates the crude rates of RA-related hand and wrist surgery across the United States. Across HRR, annual rates of surgery varied 9-fold, ranging from no surgeries performed in 4 small regions in the east or south of the United States (Lancaster, Pennsylvania; Longview, Texas; Houma, Los Angeles; and Muskegon, Michigan) to more than 80 per 10,000 patients (Ogden, Utah; Grand Forks, North Dakota; Fort Collins, Colorado). Clusters of high annual rates of RA-related hand and wrist surgery appeared in the West Coast and Central areas of the United States, and clusters of low surgical rates appeared in eastern states, such as New York, Pennsylvania, South Carolina, Georgia, and Florida.

Patient factors significantly influenced receipt of hand and wrist surgery for RA, specifically age, sex, race, and SES (Table 3). For example, patients with RA aged $\geq 80$ years were less likely to undergo hand or wrist arthroplasty and/or arthrodesis compared with patients aged 65-69 years (OR $0.29,95 \%$ CI $0.25-0.34$ ). Women were more likely to undergo arthroplasty and/or arthrodesis compared with men (OR 2.05, 95\% CI 1.85-2.28), and African American patients were less likely to undergo joint procedures compared with whites (OR $0.58,95 \%$ CI $0.48-0.70$ ). Compared with patients who lived in metropolitan counties, patients who lived in rural areas were more likely to undergo arthroplasty and/or arthrodesis (OR 1.31, 95\% CI 1.04-1.66). Finally, patients of higher SES were more likely
Table 2. Characteristics of the study cohort.

\begin{tabular}{|c|c|c|}
\hline Characteristics & No. Patients & $\%$ \\
\hline \multicolumn{3}{|l|}{ Age, yrs } \\
\hline $65-69$ & 112,252 & 32 \\
\hline $70-74$ & 89,542 & 25 \\
\hline $75-79$ & 76,155 & 21 \\
\hline$\geq 80$ & 78,112 & 22 \\
\hline \multicolumn{3}{|l|}{ Sex } \\
\hline Male & 92,595 & 26 \\
\hline Female & 263,466 & 74 \\
\hline \multicolumn{3}{|l|}{ Race } \\
\hline White & 315,407 & 89 \\
\hline African American & 26,290 & 7 \\
\hline Other & 14,364 & 4 \\
\hline \multicolumn{3}{|l|}{ SES } \\
\hline Low & 79,725 & 22 \\
\hline Medium & 96,135 & 27 \\
\hline High & 180,201 & 51 \\
\hline \multicolumn{3}{|c|}{ State assistance on Medicare premium } \\
\hline No & 319,861 & 90 \\
\hline Yes & 36,200 & 10 \\
\hline \multicolumn{3}{|l|}{ Rural status } \\
\hline Metropolitan county & 287,893 & 81 \\
\hline Non-metropolitan county & 60,340 & 17 \\
\hline Completely rural & 7828 & 2 \\
\hline \multicolumn{3}{|c|}{ Density of orthopedic surgeons ${ }^{\dagger}$} \\
\hline Low, fewer than 58 & 115,373 & 32 \\
\hline Medium, 58 to 67 & 120,331 & 34 \\
\hline High, more than 67 & 120,357 & 34 \\
\hline \multicolumn{3}{|l|}{ Density of plastic surgeons ${ }^{\dagger}$} \\
\hline Low, fewer than 15 & 117,763 & 33 \\
\hline Medium, 15 to 21 & 118,437 & 33 \\
\hline High, more than 21 & 119,861 & 34 \\
\hline \multicolumn{3}{|l|}{ Density of rheumatologists ${ }^{\dagger}$} \\
\hline Low, fewer than 9 & 117,602 & 33 \\
\hline Medium, 9 to 13 & 117,159 & 33 \\
\hline High, more than 13 & 121,300 & 34 \\
\hline \multicolumn{3}{|l|}{ Regional rate of biologics use } \\
\hline Low, $1.0 \%$ to $4.3 \%$ & 115,850 & 33 \\
\hline Medium, $4.3 \%$ to $5.6 \%$ & 120,507 & 34 \\
\hline High, $5.6 \%$ to $13.6 \%$ & 119,704 & 34 \\
\hline
\end{tabular}

\footnotetext{
$\dagger$ Density of specialists is number of specialists in each hospital referral region per 1 million residents in the region. ${ }^{\ddagger}$ Average rate of biologics between 2006 and 2010 was calculated for each hospital referral region in the United States as percent of patients with RA who received biologics therapy during the study period. SES: socioeconomic status; RA: rheumatoid arthritis.
}

to undergo arthroplasty and/or arthrodesis compared with patients of lower SES (OR 1.20, 95\% CI 1.08-1.34).

Tendon reconstruction was also influenced by patient factors. Similar to joint reconstructive procedures, younger (OR 2.63, 95\% CI 1.89-3.57), female patients (OR 1.64, 95\% CI $1.30-2.07$ ) were more likely to undergo tendon reconstruction compared with older, male patients. Additionally, African American patients (OR 0.65, 95\% CI $0.42-1.00$ ) and patients of lower SES (OR 0.71, 95\% CI $0.54-0.96)$ were less likely to undergo tendon reconstruction. Finally, patients who lived in rural areas were 


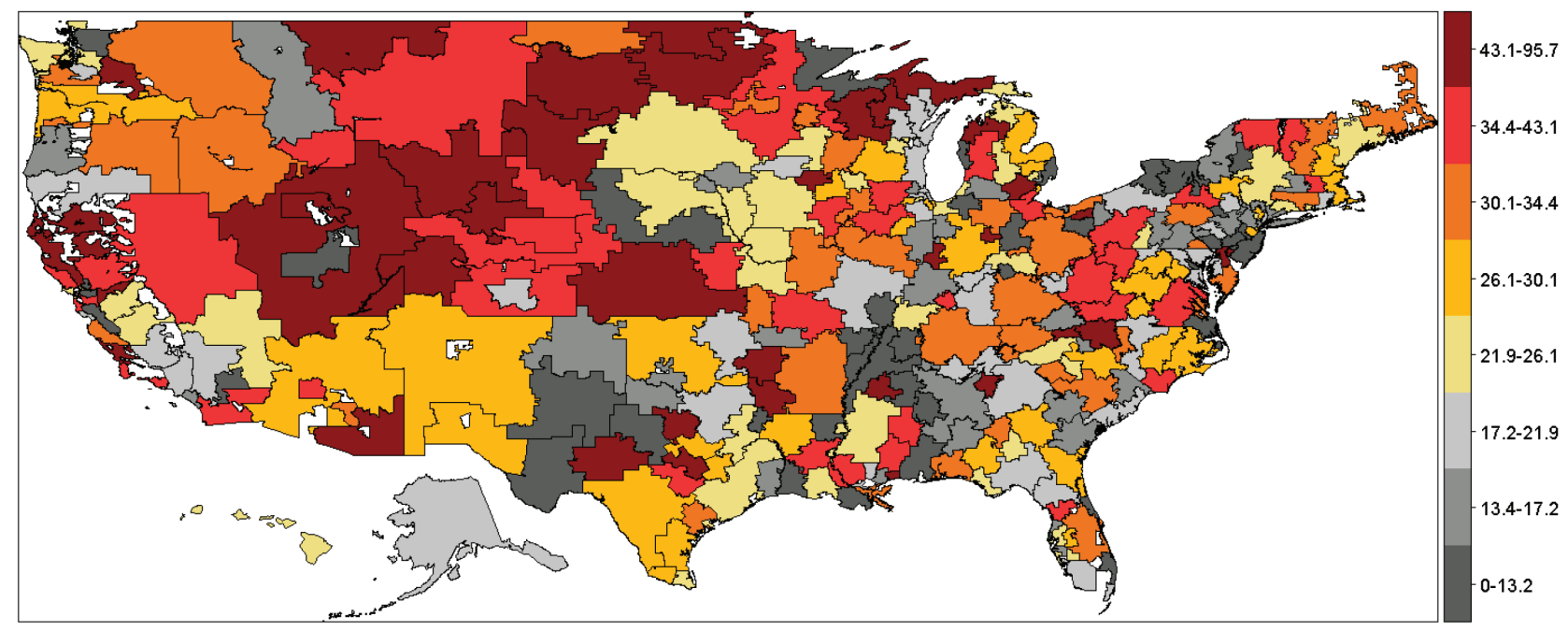

Figure 1. Annual rate of RA hand and wrist surgery (per 10,000 patients with RA) among US Medicare beneficiaries in 306 hospital referral regions, 2006-2010. RA: rheumatoid arthritis.

more likely to undergo reconstruction (OR $1.92,95 \%$ CI 1.19-3.09) compared with patients who lived in urban areas (Table 3).

We examined the effect of regional density of specialists on RA-related hand and wrist surgery. In our cohort, patients who resided in areas with a high density of orthopedic surgeons were more likely to undergo arthroplasty and/or arthrodesis (OR 1.16, 95\% CI 1.05-1.29) compared with patients who lived in areas with a lower density of orthopedic surgeons. The effect of plastic surgeon density was less uniform, and patients who lived in areas with a moderate density of plastic surgeons were more likely to undergo arthroplasty and/or arthrodesis compared with patients who lived in areas with a lower density of plastic surgeons (OR 1.10, 95\% CI 1.01-1.21). However, the same effect was not seen among patients living in areas with the highest density of plastic surgeons. Surgeon density did not influence tendon reconstruction procedures. Alternatively, regional density of rheumatologists was significantly associated with likelihood of receiving hand and wrist arthroplasty and arthrodesis among patients with RA. Patients who lived in areas with more than 13 rheumatologists/1 million residents were significantly less likely to undergo surgery (OR $0.76,95 \%$ CI $0.69-0.85$ ) compared to patients who lived in areas with fewer than 10 rheumatologists/1 million residents (Table 3). We then examined the crude and adjusted rates of arthroplasty and arthrodesis, stratified by rheumatologist density (Figure 2). Even after adjusting for other patient and regional factors, rates of surgery remained lower in areas with a higher density of rheumatologists. After controlling for all patient and provider characteristics, regional density of biologics use was not significantly correlated with rate of RA-related hand and wrist surgery.
Finally, we examined the differences in patient characteristics among individuals who resided in areas of low, medium, and high rheumatologist density (Table 4). In our cohort, age, sex, race, SES, and rural status were significantly associated with the area of residence. For example, in areas with a high density of rheumatologists, $25 \%$ of patients with RA were ages $\geq 80$ years and $29 \%$ were $65-69$ compared with lower-density areas, in which $19 \%$ of patients were $\geq 80$ years and 34\% were ages 65-69 ( $p<$ $0.001)$. Areas with a high density of rheumatologists also had significantly more metropolitan patients $(89 \%$ vs $74 \%$, $\mathrm{p}<0.001)$. Finally, higher-density areas were also associated with a greater percentage of high SES patients $(66 \%$ in high density areas vs $40 \%$ in low density areas, $\mathrm{p}<0.001$ ).

\section{DISCUSSION}

RA remains common among older individuals, afflicting over 350,000 Medicare beneficiaries from 2006 to 2010. In our study, we identified a low rate of RA-related hand and wrist reconstructive procedures among the elderly population in the United States, but found substantial variation in RA-related hand and wrist procedures driven by patient and regional factors. Patients who lived in areas with a greater number of rheumatologists were less likely to undergo RA-related hand and wrist joint fusion and replacement. Additionally, younger, female, white patients with RA and patients who resided in rural areas were more likely to undergo both arthroplasty/arthrodesis of hand and wrist, and hand tendon reconstruction. Finally, in our cohort, patients of lower SES underwent fewer surgical procedures, including both joint and tendon reconstruction. Although the reasons for this are likely multifactorial, it is possible that individuals of lower SES lack access to appropriate specialist care, have additional comorbid conditions 
Table 3. Patient characteristics of the study cohort and adjusted OR of receiving RA-related hand or wrist surgery during the study period.

\begin{tabular}{|c|c|c|c|c|}
\hline \multirow{2}{*}{ Patient Characteristics } & \multicolumn{2}{|c|}{ Arthroplasty/Arthrodesis } & \multicolumn{2}{|c|}{ Tendon Reconstructive Procedures } \\
\hline & OR $(95 \% \mathrm{CI})^{*}$ & $\mathrm{p}$ & OR $(95 \% \mathrm{CI})^{*}$ & $\mathrm{p}$ \\
\hline \multicolumn{5}{|l|}{ Age, yrs } \\
\hline $65-69$ & 1 & - & 1 & \\
\hline $70-74$ & $0.93(0.85-1.02)$ & 0.12 & $1.13(0.91-1.40)$ & 0.26 \\
\hline $75-79$ & $0.68(0.61-0.76)$ & $<0.001$ & $0.82(0.64-1.06)$ & 0.13 \\
\hline$\geq 80$ & $0.29(0.25-0.34)$ & $<0.001$ & $0.38(0.28-0.53)$ & $<0.001$ \\
\hline \multicolumn{5}{|l|}{ Sex } \\
\hline Male & 1 & - & 1 & \\
\hline Female & $2.05(1.85-2.28)$ & $<0.001$ & $1.64(1.30-2.07)$ & $<0.001$ \\
\hline \multicolumn{5}{|l|}{ Race } \\
\hline White & 1 & - & 1 & \\
\hline African American & $0.58(0.48-0.70)$ & $<0.001$ & $0.65(0.42-1.00)$ & 0.05 \\
\hline Other & $0.79(0.63-0.98)$ & 0.03 & $0.80(0.47-1.35)$ & 0.40 \\
\hline \multicolumn{5}{|l|}{ SES } \\
\hline Low & 1 & - & 1 & \\
\hline Medium & $1.15(1.03-1.29)$ & 0.01 & $1.35(1.02-1.77)$ & 0.03 \\
\hline High & $1.20(1.08-1.34)$ & 0.001 & $1.41(1.08-1.85)$ & 0.01 \\
\hline \multicolumn{5}{|c|}{ State financial assistance on Medicare premium } \\
\hline No & 1 & - & 1 & \\
\hline Yes & $1.01(0.88-1.15)$ & 0.93 & $0.79(0.55-1.13)$ & 0.19 \\
\hline \multicolumn{5}{|l|}{ Rural status } \\
\hline Metropolitan county & 1 & - & 1 & \\
\hline Non-metropolitan county & $1.13(1.02-1.26)$ & 0.02 & $0.96(0.73-1.25)$ & 0.76 \\
\hline Completely rural & $1.31(1.04-1.66)$ & 0.02 & $1.92(1.19-3.09)$ & 0.007 \\
\hline \multicolumn{5}{|l|}{ Density of orthopedic surgeons } \\
\hline Low, fewer than 58 & 1 & - & 1 & \\
\hline Medium, 58 to 67 & $1.00(0.91-1.11)$ & 0.99 & $0.87(0.69-1.10)$ & 0.26 \\
\hline High, more than 67 & $1.16(1.05-1.29)$ & 0.004 & $0.91(0.71-1.15)$ & 0.43 \\
\hline \multicolumn{5}{|l|}{ Density of plastic surgeons } \\
\hline Low, fewer than 15 & 1 & - & & \\
\hline Medium, 15 to 21 & $1.10(1.01-1.21)$ & 0.04 & $1.06(0.85-1.32)$ & 0.61 \\
\hline High, more than 21 & $0.95(0.85-1.05)$ & 0.32 & $1.05(0.82-1.33)$ & 0.71 \\
\hline \multicolumn{5}{|l|}{ Density of rheumatologists } \\
\hline Low, fewer than 9 & 1 & - & 1 & \\
\hline Medium, 9 to 13 & $0.97(0.88-1.07)$ & 0.54 & $1.10(0.88-1.38)$ & 0.41 \\
\hline High, more than 13 & $0.76(0.69-0.85)$ & $<0.001$ & $0.97(0.75-1.24)$ & 0.80 \\
\hline \multicolumn{5}{|l|}{ Regional rate of biologics use } \\
\hline Low, $1.0 \%$ to $4.3 \%$ & 1 & - & 1 & \\
\hline Medium, $4.3 \%$ to $5.6 \%$ & $0.97(0.89-1.07)$ & 0.60 & $1.11(0.88-1.38)$ & 0.38 \\
\hline High, $5.6 \%$ to $13.6 \%$ & $0.96(0.88-1.06)$ & 0.46 & $1.01(0.81-1.27)$ & 0.90 \\
\hline
\end{tabular}

* Adjusted OR of receiving hand and wrist arthroplasty/arthrodesis or tendon reconstruction during the study period, adjusting simultaneously by age, sex, race, number of comorbid conditions, SES, state financial assistance, rural status, density of specialists, and regional rate of biologics use, as well as number of years each individual stayed in the study cohort. SES: socioeconomic status; RA: rheumatoid arthritis.

precluding aggressive medical or surgical intervention, or have fewer available financial resources. Since Alderman, et $a l^{12}$ in 2002 described regional differences in rheumatoid hand and wrist reconstruction using national claims data, pronounced variation in RA treatment continues to persist. Our findings suggest the influence of providers on these differences. First, surgical rates may represent differential access to subspecialty care, particularly to rheumatologists with expertise in inflammatory arthropathies. In the United States, a relative shortage of rheumatologists exists, and many patients with RA are managed by providers who do not have subspecialty training, who may opt for less-aggressive immunosuppressive regimens, particularly among older individuals ${ }^{18}$. Individuals without access to newer DMARD may progress more quickly toward advanced deformities, resulting in higher rates of surgical therapy among those patients treated in areas with fewer rheumatologists. Additionally, our findings may represent differences in referral patterns for surgical intervention. Rheumatologists are often skeptical of the effectiveness of surgical interventions for RA, which influences referral rates for extremity surgery. Lower rates of surgery in some areas may reflect not only differences in aggressiveness of medical therapy, but also differences in treatment preferences ${ }^{19,20}$.

The majority of patients are referred to rheumatologists by primary care physicians following the diagnosis of RA, 


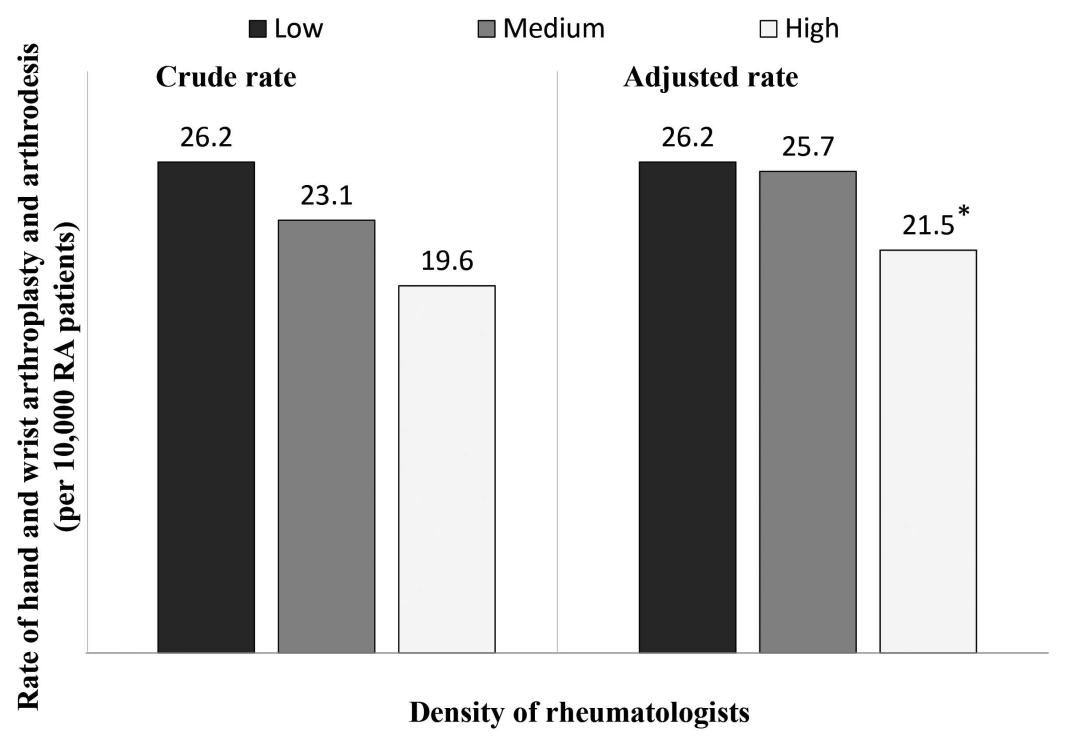

Figure 2. Crude and adjusted rate of RA-related hand and wrist surgery among Medicare beneficiaries who lived in areas of different quartiles of rheumatologist density. $* p<0.05$, from multivariate logistic regression model, adjusting simultaneously by all patient characteristics and number of years in study cohort. RA: rheumatoid arthritis.

Table 4. Distribution of baseline patient characteristics by density of rheumatologists of the hospital referral region. Values are \% unless otherwise specified.

\begin{tabular}{lcccc}
\hline \multirow{2}{*}{ Patient Characteristics } & \multicolumn{5}{c}{ Density of Rheumatologists } & p \\
& Low & Medium & High & \\
& & & & \\
\hline Age, yrs & 34 & 32 & 29 & $<0.001$ \\
$\quad 65-69$ & 26 & 25 & 24 & \\
$70-74$ & 21 & 21 & 22 & \\
$75-79$ & 19 & 22 & 25 & \\
$\quad$ 80 & & & & \\
Sex & 27 & 26 & 25 & $<0.001$ \\
$\quad$ Male & 73 & 74 & 75 & \\
$\quad$ Female & & & & \\
Race & 90 & 87 & 88 & $<0.001$ \\
$\quad$ White & 6 & 7 & 8 & \\
African American & 3 & 5 & 4 & \\
$\quad$ Other & & & & \\
SES & 25 & 20 & 11 & $<0.001$ \\
$\quad$ Low & 31 & 29 & 21 & \\
$\quad$ Medium & 40 & 48 & 66 & \\
$\quad$ High & 4 & 4 & 2 & \\
$\quad$ Missing & & & & \\
Rural status & 74 & 80 & 89 & $<0.001$ \\
$\quad$ Metropolitan county & 22 & 18 & 9 & \\
$\quad$ Non-metropolitan county & 22 & 2 & 1 & \\
Completely rural & 3 & & & \\
\hline
\end{tabular}

SES: socioeconomic status.

and surgeons are rarely the initial treatment provider. In the United States, about $70 \%$ of hand surgeons are trained in orthopedic surgery, $20 \%$ in plastic surgery, and $10 \%$ in general surgery ${ }^{21}$. In our study, we identified a significant difference in rates of surgery by density of orthopedic surgeons. Surgeons often perceive that medical therapy is extended into advanced stages of RA, making it impossible to reconstruct functionally and aesthetically acceptable hands ${ }^{12,19}$. To date, 1 of the largest prospective cohort studies of patients with rheumatoid hand deformities revealed that patients with RA treated with silicone metacarpophalangeal arthroplasty continue to experience longterm improvement in hand function and appearance compared with patients managed medically. Therefore, despite this controversy, surgery remains an important option to relieve pain and improve function, hand appearance, and quality of life among patients with $\mathrm{RA}^{22}$.

Geographic variation in care is common across the United States ${ }^{23,24,25}$. Although such variation is thought to represent differences in quality of care, the reasons for this are multifactorial ${ }^{23,26}$. Our analysis in Figure 1 represents the unadjusted rates across HRR in the United States. Our multivariate analysis does highlight several patient factors, such as age and sex, and regional factors, such as rheumatologist density, that explain the variation in rates. However, collectively, these factors explain only $0.3 \%$ of the variation, and unmeasured factors certainly exist. For example, our study lacks detail in clinical factors that may influence surgery, such as disease severity, and does not identify patient preferences for treatment ${ }^{19}$. It is also plausible that there is underlying geographic variation in the incidence of RA. An analysis of the Nurses' Health Study revealed an increased incidence of RA among women in New England and the Midwest ${ }^{27}$. Although the etiology of RA remains unknown, there is likely regional variation in risk factors, 
such as genetic pools or environmental factors that have yet to be identified ${ }^{28}$.

Our study has several notable limitations. First, administrative data were subject to systematic coding errors and were not collected with the explicit purpose of examining practice patterns. However, we required at least 2 claims, including an RA diagnosis, to ensure the validity of our selected cohort, and a study comparing diagnoses between office records and Medicare claimed to have found a $90 \%$ correlation between diagnoses ${ }^{29}$. Owing to the stringency of our criteria, it was possible that patients with RA with limited access to care could be excluded from the cohort. Additionally, we excluded Medicare Part C enrollees (16-24\% of all Medicare beneficiaries) because their outpatient services may not be found in administrative claims data. Our study sample included only adults aged $\geq 65$ years, and our findings may not have been generalizable to younger individuals with $\mathrm{RA}^{30}$. Finally, we were not able to examine the effect of disease severity or chronicity on surgical rates, given the lack of granular detail of claims data at this level. Without clinical data, such as laboratory findings, symptomatology, and radiographic progression, we could not account for the influence of disease severity on rates of surgery. Nonetheless, Medicare data have been widely used to examine treatment patterns and provide robust data because of large, population-based samples. Compared with clinical trials or observational registries that are biased toward tertiary care centers, CMS claims data reflect real-world clinical practice $29,31,32,33$.

Despite these limitations, our findings reveal significant variation in RA treatment pattern by availability of rheumatologists, and highlight important areas for future investigation. First, access to appropriate healthcare resources has been central to healthcare reform initiatives in the United States. Several interventions, such as the expansion of prescription drug coverage through Medicare Part D and the expansion of Medicaid (low-income) coverage from the Patient Protection and Affordable Care Act, may minimize disparities in access to care for chronic diseases. For example, the initiation of Medicare Part D coverage of biologic DMARD therapy for RA has improved use among vulnerable groups ${ }^{34,35}$. Additionally, healthcare reform initiatives have included specific provisions for comparative effectiveness research. Given the controversy that remains regarding the effectiveness of medical and surgical therapy for rheumatoid hand deformities, future studies that directly compare the effectiveness of surgical therapy in the setting of advanced medical therapies, such as biologic DMARD, may allow for better integration of treatment options between rheumatologists and surgeons.

Surgical reconstruction of rheumatoid hand deformities varies across the United States because of the availability of rheumatologists and patient characteristics. Future efforts that directly examine access to healthcare services, surgery referral pattern, and effectiveness of medical and surgical treatment options, as well as health policy interventions aimed at improving access to care, can minimize unwarranted variation in the management of rheumatoid hand and wrist deformities.

\section{REFERENCES}

1. Myasoedova E, Crowson CS, Kremers HM, Therneau TM, Gabriel SE. Is the incidence of rheumatoid arthritis rising?: results from Olmsted County, Minnesota, 1955-2007. Arthritis Rheum 2010;62:1576-82.

2. Helmick CG, Felson DT, Lawrence RC, Gabriel S, Hirsch R, Kwoh $\mathrm{CK}$, et al. Estimates of the prevalence of arthritis and other rheumatic conditions in the United States. Part I. Arthritis Rheum 2008;58:15-25.

3. Chung KC, Burke FD, Wilgis EF, Regan M, Kim HM, Fox DA. A prospective study comparing outcomes after reconstruction in rheumatoid arthritis patients with severe ulnar drift deformities. Plast Reconstr Surg 2009;123:1769-77.

4. Chung KC, Kotsis SV, Kim HM. A prospective outcomes study of Swanson metacarpophalangeal joint arthroplasty for the rheumatoid hand. J Hand Surg Am 2004;29:646-53.

5. Waljee JF, Chung KC. Objective functional outcomes and patient satisfaction after silicone metacarpophalangeal arthroplasty for rheumatoid arthritis. J Hand Surg Am 2012;37:47-54.

6. Weiss R, Ehlin A, Montgomery SM, Wick M, Stark A, Wretenberg P. Decrease of RA-related orthopaedic surgery of the upper limbs between 1998 and 2004: data from 54,579 Swedish RA inpatients. Rheumatology 2008;47:491-4.

7. Fevang BT, Lie SA, Havelin LI, Engasaeter LB, Furnes O. Reduction in orthopedic surgery among patients with chronic inflammatory joint disease in Norway, 1994-2004. Arthritis Rheum 2004;57:529-32.

8. Momohara S, Ikari K, Mochizuki T, Kawamura K, Tsukahara S, Toki $\mathrm{H}$, et al. Declining use of synovectomy surgery for patients with rheumatoid arthritis in Japan. Ann Rheum Dis 2009;68:291-2.

9. Nikiphorou E, Carpenter L, Morris S, MacGregor AJ, Dixey J, Kiely P, et al. Hand and foot surgery rates in rheumatoid arthritis have declined from 1986 to 2011, but large-joint replacement rates remain unchanged: results from two UK inception cohorts. Arthritis Rheumatol 2014;66:1081-9.

10. Korpela M, Laasonen L, Hannonen P, Kautiainen H, Leirisalo-Repo M, Hakala M, et al. Retardation of joint damage in patients with early rheumatoid arthritis by initial aggressive treatment with disease-modifying antirheumatic drugs: five-year experience from the FIN-RACo study. Arthritis Rheum 2004;50:2072-81.

11. Alderman AK, Ubel PA, Kim HM, Fox DA, Chung KC. Surgical management of the rheumatoid hand: consensus and controversy among rheumatologists and hand surgeons. J Rheumatol 2003;30:1464-72.

12. Alderman AK, Chung KC, Demonner S, Spilson SV, Hayward RA The rheumatoid hand: a predictable disease with unpredictable surgical practice patterns. Arthritis Rheum 2002;47:537-42.

13. Zhang J, Xie F, Delzell E, Chen L, Kilgore ML, Yun H, et al. Trends in the use of biologic agents among rheumatoid arthritis patients enrolled in the US medicare program. Arthritis Care Res 2013;65:1743-51.

14. Diez Roux AV, Merkin SS, Arnett D, Chambless L, Massing M, Nieto FJ, et al. Neighborhood of residence and incidence of coronary heart disease. N Engl J Med 2001;345:99-106.

15. Elixhauser A, Steiner C, Harris DR, Coffey RM. Comorbidity measures for use with administrative data. Med Care 1998;36:8-27.

16. Butler MA, Beale CL. Rural-urban continuum codes for metro and nonmetro counties, 1993. Washington: United States Department of 
Agriculture, Economic Research Service; 1994.

17. Wennberg JE, Cooper M. The Dartmouth atlas of health care. Chicago: American Hospital Publishing Chicago; 1996.

18. Deal CL, Hooker R, Harrington T, Birnbaum N, Hogan P, Bouchery E, et al. The United States rheumatology workforce: supply and demand, 2005-2025. Arthritis Rheum 2007;56:722-9.

19. Alderman AK, Chung KC, Kim HM, Fox DA, Ubel PA. Effectiveness of rheumatoid hand surgery: contrasting perceptions of hand surgeons and rheumatologists. J Hand Surg Am 2003; 28:3-11.

20. Burke FD, Miranda SM, Owen VM, Bradley MJ, Sinha S. Rheumatoid hand surgery: differing perceptions amongst surgeons, rheumatologists and therapists in the UK. J Hand Surg Eur Vol 2011;36:632-41.

21. Brunworth LS, Chintalapani SR, Gray RR, Cardoso R, Owens PW. Resident selection of Hand Surgery Fellowships: a survey of the 2011, 2012, and 2013 Hand Fellowship graduates. Hand 2013;8:164-71.

22. Chung KC, Burns PB, Kim HM, Burke FD, Wilgis EF, Fox DA. Long-term followup for rheumatoid arthritis patients in a multicenter outcomes study of silicone metacarpophalangeal joint arthroplasty. Arthritis Care Res 2012;64:1292-300.

23. Weinstein JN, Birkmeyer JD. The Dartmouth atlas of musculoskeletal health care. Chicago: American Hospital Association Press; 2000.

24. Weinstein JN, Lurie JD, Olson PR, Bronner KK, Fisher ES. United States' trends and regional variations in lumbar spine surgery: 1992-2003. Spine 2006;31:2707-14.

25. Rhodes LA, Tan AL, Tanner SF, Radjenovic A, Hensor EM, Reece $\mathrm{R}$, et al. Regional variation and differential response to therapy for knee synovitis adjacent to the cartilage-pannus junction and suprapatellar pouch in inflammatory arthritis: implications for pathogenesis and treatment. Arthritis Rheum 2004;50:2428-32.

26. Skinner J, Weinstein JN, Sporer SM, Wennberg JE. Racial, ethnic, and geographic disparities in rates of knee arthroplasty among Medicare patients. N Engl J Med 2003;349:1350-9.

27. Costenbader KH, Chang SC, Laden F, Puett R, Karlson EW. Geographic variation in rheumatoid arthritis incidence among women in the United States. Arch Intern Med 2008;168:1664-70.

28. Edwards CJ, Campbell J, van Staa T, Arden NK. Regional and temporal variation in the treatment of rheumatoid arthritis across the UK: a descriptive register-based cohort study. BMJ Open 2012;2.
29. Katz JN, Barrett J, Liang MH, Bacon AM, Kaplan H, Kieval RI, et al. Sensitivity and positive predictive value of Medicare Part B physician claims for rheumatologic diagnoses and procedures. Arthritis Rheum 1997;40:1594-600.

30. Tutuncu Z, Reed G, Kremer J, Kavanaugh A. Do patients with older-onset rheumatoid arthritis receive less aggressive treatment? Ann Rheum Dis 2006;65:1226-9.

31. Lauderdale DS, Furner SE, Miles TP, Goldberg J. Epidemiologic uses of Medicare data. Epidemiol Rev 1993;15:319-27.

32. Wailoo AJ, Bansback N, Brennan A, Michaud K, Nixon RM, Wolfe F. Biologic drugs for rheumatoid arthritis in the Medicare program: a cost-effectiveness analysis. Arthritis Rheum 2008;58:939-46.

33. Solomon DH, Yelin E, Katz JN, Lu B, Shaykevich T, Ayanian JZ. Treatment of rheumatoid arthritis in the Medicare Current Beneficiary Survey. Arthritis Res Ther 2013;15:R43.

34. Polinski JM, Mohr PE, Johnson L. Impact of Medicare Part D on access to and cost sharing for specialty biologic medications for beneficiaries with rheumatoid arthritis. Arthritis Rheum 2009;61:745-54

35. Doshi JA, Li P, Puig A. Impact of the Medicare Modernization Act of 2003 on utilization and spending for medicare part B-covered biologics in rheumatoid arthritis. Arthritis Care Res 2010;62:354-61.

APPENDIX 1. Healthcare Common Procedure Codes and generic name of biologic DMARD.

\begin{tabular}{lc}
\hline Generic Name & Healthcare Common Procedure Codes \\
\hline Abatacept/maltose & $\mathrm{J} 0219$ \\
Adalimumab & $\mathrm{J} 0135$ \\
Anakinra & - \\
Certolizumab pegol & $\mathrm{J} 0718$ \\
Etanercept & $\mathrm{J} 1438$ \\
Golimumab & - \\
Infliximab & $\mathrm{J} 1475$ \\
Rituximab & $\mathrm{J} 9310$ \\
Tocilizumab & - \\
Tofacitinib & - \\
\hline
\end{tabular}

DMARD: disease-modifying antirheumatic drug. 